The next day (the 18th) he was still very gravely ill, his temperature being down to $95^{\circ} \mathrm{F}$. and his pulse up to 150 , thready and barely palpable. He vomited altered blood, the vomiting being effortless. A duodenal tube was passed and was left in situ. The output of urine fell almost to zero, and he was pale, grey, and sweating, and looked like dying. The oedema had spread up from the thigh on to the abdomen, back, and lower part of chest. In the evening, as a last resort, multiple incisions were made in the thigh, back, and abdominal and chest walls. Considerable quantities of serum and a little gas escaped. A saline-sulphathiazole drip was started-3/4 pint in four hours. On the 20th he was still ill, but his pulse had fallen from 140 to 108 and its volume was better. His temperature had risen from $95^{\circ}$ to $98^{\circ} \mathrm{F}$. and the amount of urine excreted had increased. In addition to getting whole blood he was having intravenous saline and dextrose most of the time. A swab taken on the 22 nd was sterile and the patient continued to improve, his chest being clear and free from pulmonary oedema. The next day he developed a mild phlebitis of the left leg. The drip was discontinued.

During the following week he received two pints of fresh blood and polyvalent A.G.G.S. The penicillin drip was continued. On the 27 th his haemoglobin content was $59 \%$. All this time there was a copious serous discharge from the wounds, and precautions were taken to give plenty of fluids by mouth or intravenously. The serum protein was investigated, and showed total protein $5.40 \%$-albumin $3.12 \%$, globulin $2.28 \%$ (by difference). An area of redness and swelling developed on the inner side of the right thigh, so on July 30 more multiple incisions were made, but only blood and serum exuded. This process was repeated a few days later. The swelling of the thigh and leg gradually subsided. On the 31st his haemoglobin had fallen to $52 \%$, so more fresh blood was given, and it rose to $58 \%$ by the next day. A swab showed scanty Staph. aureus, enterococci, coliforms, and Gram-positive bacilli. A swab taken three days later revealed Gram-positive cocci, scanty Gram-positive bacilli-probably anthracoids-and some resistant staphylococci and enterococci.

By Aug. 6 his haemoglobin was $65 \%$ and he continued to improve. The serous discharge from the wounds diminished-in fact, most of the wounds were now healed; but there was a discharge of pus containing bubbles of gas from a few of the wounds above and below the original shell wound and the thigh was distinctly resonant on percussion in these situations. It was decided to operate again, and on Aug. 25 the wounds were opened up and from each site, above and below the original wound, a large grey-black spongy slough was removed. The pathological report on these specimens was: "The material consists of two masses of soft friable tissue $5 \times 2.5 \times$ $1 \mathrm{~cm}$. and $9 \times 5 \times 1.5 \mathrm{~cm}$. The material is necrotic, and muscle is not recognized. Microscopically this is completely necrotic. Adipose tissue is the only recognizable one in the section." The patient improved rapidly after this; the wounds (operation and shell) healed completely and he was allowed up. He was discharged to his home on Sept. 28. All the movements of the limb were complete, but there was, of course, wasting of the muscles and he walked with a limp. His general condition was good.

During the course of his illness more than three dozen incisions were made in the thigh, back, abdomen, and chest. He had been given nine pints $(900,000$ units) of penicillin solution intramuscularly and about 150,000 units of anti-gas-gangrene serum as well as a second full course of sulphonamides. In addition he had large quantities of blood, plasma, saline, and dextrose intravenously.

I acknowledge my indebtedness to the resident medical officer of the Royal Victoria Infirmary, Dr. J. H. Craig, who was responsible for the intravenous therapy and whose interest in the case contributed largely to the happy ending.

The latest report of Dr. H. P. Newsholme as medical officer of health for the City of Birmingham shows that the year 1943 gave cause for satisfaction in a number of aspects of the public health. New records were reached in an unusually large number of directions. The year saw the lowest infant death rate ever recorded in the city; and, with this, the lowest illegitimate infant mortality, falling actually below the particularly low general infant mortality. The city recorded its lowest neonatal death rate for infants under 1 month of age, and its lowest stillbirth rate. The year showed also the lowest notification rate of non-pulmonary tuberculosis so far recorded in Birmingham; last, but not least, the lowest maternal death rate ever recorded in the city was reached in 1943. Against these pleasant facts Dr. Newsholme notes that, largely as a result of a widespread epidemic of influenza, the death rate did not drop quite to the low level of 1942 ; that there was a $35 \%$ increase in the number of new cases of syphilis coming up for clinic treatment ; and that there was some increase, though not a large one, in new cases of tuberculosis of the lungs in 1943. No substantial change occurred during the year in the housing position, and the problem of overcrowding as well as that of families housed in dilapidated property remains very serious.

\section{TWO CASES OF TETANUS BY}

LESLIE COLE, M.D., F.R.C.P.

Honorary Physician, Addenbrooke's Hospital, Cambridge

The first of these cases of tetanus is described because it is an interesting example of severe local tetanus developing during the course of a mild generalized attack; and the second -a straightforward example of severe tetanus-because it illustrates certain special points in treatment.

Case I

On Aug. 8,1944 , the patient, a boy aged $5 \frac{1}{2}$, trod on a rusty nail, injuring the left foot. No special treatment was given, and the wound appeared to heal satisfactorily. On the evening of Aug. 11 he seemed generally out of sorts and complained of abdominal pain, which woke him up every hour throughout the night. In the morning he had difficulty in opening his mouth. He was admitted to Addenbrooke's Hospital on Aug. 12, about 16 hours after the onset, and then showed the characteristic signs of tetanus: trismus, risus sardonicus, stiffness, opisthotonos, and abdominal rigidity. The foot and leg on the injured side were slightly stiffer than on the other. On the ball of the left big toe was an almost healed wound with no sign of sepsis. There were no other wounds.

Treatment and Prognosis.-200,000 units of antitoxin were given at once, mainly by the intravenous route, and during the next few days trismus and rigidity of the trunk increased. On the 13th reflex excitability increased, and on the 14th he had some slight generalized spasms. Synchronously with the development of these general signs, the rigidity of the left leg became steadily more severe, and by the 14th the whole leg, in contrast to the other limbs, was in continuous muscular spasm and so stiff and rigid that to produce even slight movement at the knee- and ankle-joints required the exercise of considerable force. The leg was slightly flexed at the knee, with the foot plantar-flexed and inverted (see Fig.). Very slight reflex spasms



CASE I.-Local tetanus, left leg and foot.

occurred several times daily until the 17 th, and tonic rigidity of the trunk and trismus until the 31st. A severe degree of stiffness of the left leg remained throughout the whole illness, and the left foot was still rather stiff on Sept. 9, when the patient was able to walk about. All stiffness, both local and general, had passed off. when he was discharged on Sept. 25.

At no stage of the illness were the spasms severe, and $10 \mathrm{gr}$. of potassium bromide four-hourly and $1 \frac{1}{2} \mathrm{dr}$. of paraldehyde per rectum once nightly for the first five days were sufficient to promote sleep.

\section{Case II}

On Sept. 17 a boy aged 8 fell off his bicycle, grazing the dorsum of his right foot. This was treated with dettol and bound up, but the wound was not kept very clean. On the evening before admission he felt generally out of sorts and complained of severe backache and difficulty in opening his mouth. These symptoms became rapidly more severe, and he was admitted to hospital on Sept. 27. On admission risus sardonicus and trismus were pronounced, and he could only separate the teeth a quarter of an inch. There was also severe rigidity of the whole trunk with slight opisthotonos. There were a partly healed septic abrasion on the right external malleolus and other scabs on the right heel.

Treatment and Prognosis.-On admission 100,000 units of antitoxin were given intravenously, and the wound was subsequently cleaned and scabs were removed. The signs increased rapidly in severity, and 24 hours after the onset reflex excitability was becoming extremely marked, and, 36 hours after, generalized convulsions were occurring regularly every half-hour, with very severe trismus. Other ominous signs were a rising pulse rate and temperature and inability to take food because of the severity of the trismus. There was also considerable difficulty with micturition. 
Both rectal paraldehyde and avertin in basal anaesthetic doses were first tried to control the spasms, but were unsatisfactory because they were not retained. Pentothal, in doses of $0.25 \mathrm{~g}$. intravenously, was then used as a temporary expedient, but was not very successful. Intramuscular paraldehyde in doses of $3 \mathrm{c.cm}$. was now tried, and as it proved extremely effective it was continued as required for the control of spasms until Oct. 6. For the first few days a dose of 2 or $3 \mathrm{c} . \mathrm{cm}$. was given twice every 24 hours, and later once. To combat the increasing dehydration, an intravenous drip was started on Sept. 28 and was continued for 48 hours, the needle being kept in the vein by a plaster-of-Paris splint to the forearm.

On Sept. 29 the temperature rose to $103^{\circ}$, the pulse to 160 , and the respirations to 45 . The chest was very bubbly, cyanosis was present, and it was clear that pneumonia would be likely to supervene. Sulphathiazole was therefore given, first by drip and afterwards by mouth, until Oct. 5, to a total of $26 \mathrm{~g}$. The child's condition, which was critical, showed slight improvement on Oct. 2, reflex convulsions became easier to control, and he was able to take fluids by the mouth. His condition then gradually improved, reflex spasms having ceased by Oct. 8 and the tonic rigidity by Oct. 25 . He was discharged well on Oct. 30 .

\section{Comment}

Local tetanus occurs in mild cases most commonly after prophylactic antitoxin has been given. Usually the local signs occur in the injured limb before any generalized symptoms appear. In head wounds, paralysis or spasm of cranial nerves, particularly the 7 th and sometimes the 3rd, 6th, and 9th, is another manifestation of local tetanus. It is an important warning that generalized tetanus will almost certainly develop and an indication for giving antitoxin with the utmost dispatch without waiting for trismus or other generalized signs to appear. The work of Abel et al. (1938) suggests that local tetanus is caused by toxin absorbed from the wound affecting the motor nerve endings locally before toxin carried round in the blood stream has had time to produce its effect on the motor nerves generally or to involve the synapses in the brain and spinal cord.

In Case I the signs of local tetanus in the wounded limb were very slight when the signs of generalized tetanus were marked, and it was only after antitoxin had been given that the local tetanus developed to an intense degree, persisting throughout the whole illness and even longer than the signs of tetanus in other parts of the body. It is perhaps worth noting that local tetanus of the type described in this case probably. occurs without any detectable signs or symptoms of generalized tetanus later. The following is an example of such a case.

An agricultural labourer aged 55 attended the out-patient department complaining of stiffness and rigidity of the left arm from fingers to shoulder. There was very little pain. Over his hand and forearm were numerous small scabs, which appeared to be some weeks old. The condition had come on gradially over a period of about a fortnight and passed off in the same time. There is a strong probability that this was an example of local tetanus in which the absorption of toxin from very slight wounds had been too small in amount to produce any generalized symptoms. The diagnosis in this case could not be confirmed because the scabs had dried up by the time the patient was seen.

Case II is an example of severe tetanus with a period of onset of not more than 36 hours. The whole march of the disease in its early stages suggested a very bad prognosis. The case is described to illustrate the use of three measures which proved particularly helpful in treatment-namely, (1) intramuscular paraldehyde to control reflex convulsions and promote sleep; (2) a plaster-of-Paris bandage to keep the needle of the intravenous drip in the vein during the period when convulsions were frequent and severe; (3) the early administration of sulphathiazole when the possibility of lung infection was threatening. For the first two suggestions I am indebted to my house-physician, Dr. E. Counsell.

\section{REFERENCE}

Abel, J. J., Warfield, M. F., and Chalian, W. (1938). Johns Hopk. Hosp. Bull., 63,373

The Therapeutic Research Corporation of Great Britain has elected the following officers for the year 1945: Chairman, Board of Directors, Lord Trent (Boots Pure Drug Co. Ltd.); Deputy Chairman, Dr. F. H. Carr (British Drug Houses Ltd.). Chairman, Research Panel, Mr. F. A. Robinson (Glaxo Laboratories Ltd.); Deputy Chairman, Dr. C. H. Kellaway (Wellcome Foundation Ltd.).

\section{TIBIAL BONE-MARROW TRANSFUSIONS IN INFANTS}

\author{
BY
}

\author{
F. W. GUNZ, M.D., M.R.C.P.
}

Assistant Clinical Pathologist, Addenbrooke's Hospital, Cambridge AND

\section{R. F. A. DEAN, M.R.C.S., L.R.C.P.}

House-physician, Addenbrooke's Hospital

This paper records our experience with transfusions into the marrow cavity of the tibia in small children, using the apparatus described by Janet Gimson (1944). We have employed this method of administering fluids as an alternative to intravenous therapy during the past 9 months, and are now setting out details of 35 consecutive transfusions. The total number of patients was smaller, as more than one transfusion was given to the same child in several instances. Nearly all the patients were suffering from dehydration due to gastro-enteritis.

\section{Notes on Technique}

In this series we have used the technique described by Gimson, and it will be sufficient merely to enumerate some points in which we have deviated from her technique.

The legs are most satisfactorily held by firmly bandaging the soles of the feet to a padded splint so that they are about 9 in. apart, the ends of the splint being tied to the sides of the cot. This allows some movement of the legs but prevents the needle from being dislodged; and nursing is made easier because the splint is well away from the napkin area. Full surgical precautions-mask, sterile gown, and gloves-are taken against sepsis, and the skin is cleaned several times with spirit, then covered with sterile gauze until the needle is inserted. The choice of needle is not always easy, because of the difficulty of judging the thickness of the subcutaneous tissues and of the tibia itself. The most common mistake is to use too large, not too small a needle.

The site chosen is on the flat subcutaneous surface of the tibia, at least 1 in. below the level of the tibial tuberosity. Repeated post-mortem examination showed that even in the smallest infants there is a wide marrow cavity extending almost half-way down the tibial shaft, and it is reasonable to avoid the epiphysial region by a wide margin. No local anaesthetic is used for the insertion of the transfusion needle. The trauma inflicted by the needle seems to be very little more than that occasioned by an injection of novocain, and the pain is purely momentary.

The adapter connecting the needle to the giving set is not entirely satisfactory. It would seem to need redesigning, as in its present form the junction with the needle is easily loosened and will sometimes leak in an annoying way. We have used several adapters which we made from cork and rubber tubing, and have found them quite satisfactory. A heat-cradle is useful in at least two ways: it keeps the bedclothes from touching the needle and its tubing, and can be used to warm up the leg if the drip runs slowly.

\section{The Transfusion}

Saline, glucose-saline, Hartmann's solution, and half-strength serum have been given with good results. The rate of flow is usually fast enough to meet the child's total fluid needs, but we have used transfusions more often merely to supplement fluid taken by mouth. The amounts given are shown in the accompanying Table. It was soon found that the drip usually needed slowing to prevent the development of local oedema. The unrestricted rate of flow bears a fairly direct relation to the weight of the child and to the degree of dehydration, the fluid running faster with the heavier child and the greater dehydration, and slowing down automatically as body fluids become replenished. This is in contrast to intravenous transfusion, in which the rate tends to be unpredictable. The cause for this difference seems to be-that, whereas in intravenous transfusions the rate of flow is determined by the height of the hydrostatic pressure and the size of the lumen of the vein, the ease of absorption of the 